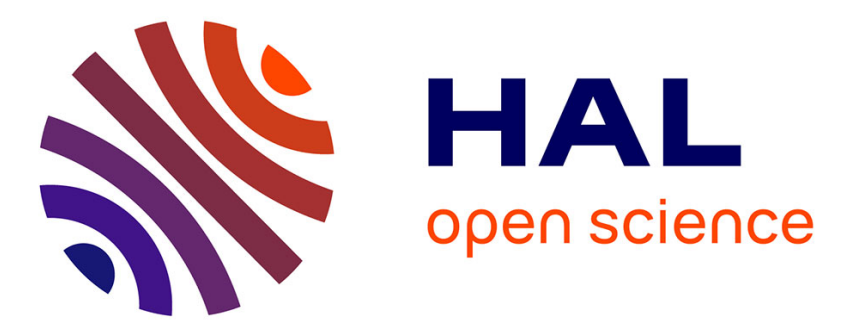

\title{
Lyapunov-based hybrid loops for stability and performance of continuous-time control systems
}

Christophe Prieur, Sophie Tarbouriech, Luca Zaccarian

\section{To cite this version:}

Christophe Prieur, Sophie Tarbouriech, Luca Zaccarian. Lyapunov-based hybrid loops for stability and performance of continuous-time control systems. Automatica, 2013, 49 (2), pp.577-584. 10.1016/j.automatica.2012.11.030 . hal-00781061

\section{HAL Id: hal-00781061 https://hal.science/hal-00781061}

Submitted on 25 Jan 2013

HAL is a multi-disciplinary open access archive for the deposit and dissemination of scientific research documents, whether they are published or not. The documents may come from teaching and research institutions in France or abroad, or from public or private research centers.
L'archive ouverte pluridisciplinaire HAL, est destinée au dépôt et à la diffusion de documents scientifiques de niveau recherche, publiés ou non, émanant des établissements d'enseignement et de recherche français ou étrangers, des laboratoires publics ou privés. 


\title{
Lyapunov-based hybrid loops for stability and performance of continuous-time control systems
}

\author{
Christophe Prieur ${ }^{\mathrm{a}, 1} \quad$ Sophie Tarbouriech ${ }^{\mathrm{b}, 1} \quad$ Luca Zaccarian $^{\mathrm{b}, 1}$ \\ ${ }^{a}$ Gipsa-lab, Grenoble Campus, 11 rue des Mathématiques, BP 46, 38402 Saint Martin d'Hères Cedex, France \\ ${ }^{\mathrm{b}}$ CNRS, LAAS, 7 avenue du colonel Roche, F-31400 Toulouse, France \\ Univ de Toulouse, LAAS, F-31400 Toulouse, France.
}

\begin{abstract}
We construct hybrid loops that augment continuous-time control systems. We consider a continuous-time nonlinear plant in feedback with a (possibly non stabilizing) given nonlinear dynamic continuous-time state feedback controller. The arising hybrid closed loops are guaranteed to follow the underlying continuous-time closed-loop dynamics when flowing and to jump in suitable regions of the closed-loop state space to guarantee that a positive definite function $V$ of the closed-loop state and/or a positive definite function $V_{p}$ of the plant-only state is non-increasing along the hybrid trajectories. Sufficient conditions for the construction of these hybrid loops are given for the nonlinear case and then specialized for the linear case with the use of quadratic functions. For the linear case we illustrate specific choices of the functions $V$ and $V_{p}$ which allow for the reduction of the overshoot of a scalar output. The proposed approaches are illustrated on linear and nonlinear examples.
\end{abstract}

Key words: hybrid systems, performance, reset controllers, asymptotic controllability, detectability, Lyapunov methods

\section{Introduction}

For a large class of nonlinear control systems which follow a purely continuous dynamics, it may be useful to consider dynamic controllers having a mixed discrete/continuous dynamics. This leads to the class of hybrid control laws which has been proven to relax certain limitations of continuous-time controllers. Among other things, hybrid controllers are also instrumental to improve the performance for nonlinear systems in the presence of disturbances. See Prieur and Astolfi (2003) for the non-holonomic integrator, and Sanfelice et al. (2006) for the inverted pendulum to focus on applications only. Also for linear plants, hybrid controllers can be fruitful. See Beker et al. (2001) for an example of a reset controller overcoming intrinsic limitations of linear control schemes. See also Beker et al. (2004); Nešić et al. (2008) where reset controllers are used to decrease the $\mathcal{L}_{2}$ gain between perturbations and the output. Consider also Aangenent et al. (2010) where it is shown that reset con-

\footnotetext{
1 Work supported by the ANR project ArHyCo, ARPEGE, contract number ANR-2008 SEGI 004 0130011459 , and by HYCON2 Network of Excellence "Highly-Complex and Networked Control Systems", grant agreement 257462. christophe.prieur@gipsa-lab.fr sophie.tarbouriech@laas.fr zaccarian@laas.fr
}

trollers may be useful to improve the $\mathcal{L}_{2}$ or $\mathcal{H}_{2}$ stability of linear systems. Finally, see Lazar and Heemels (2009) for the design of predictive controllers for the input-tostate stability of hybrid systems.

In this paper we consider a nonlinear plant:

$$
\dot{x}_{p}=\bar{f}_{p}\left(x_{p}, u\right)
$$

with $x_{p}$ in $\mathbb{R}^{n_{p}}$, in feedback interconnection with a (not necessarily stabilizing) dynamic controller:

$$
\dot{x}_{c}=\bar{f}_{c}\left(x_{c}, x_{p}\right), \quad u=\bar{h}_{c}\left(x_{c}, x_{p}\right),
$$

with $x_{c}$ in $\mathbb{R}^{n_{c}}$. Then defining the closed-loop functions $f_{p}\left(x_{p}, x_{c}\right)=\bar{f}_{p}\left(x_{p}, \bar{h}_{c}\left(x_{c}, x_{p}\right)\right)$ and $f_{c}\left(x_{p}, x_{c}\right)=$ $\bar{f}_{c}\left(x_{c}, x_{p}\right)$, the interconnection between (1) and (2) can be described in a compact way as:

$$
\frac{d}{d t}\left(x_{p}, x_{c}\right)=\left(f_{p}\left(x_{p}, x_{c}\right), f_{c}\left(x_{c}, x_{p}\right)\right)
$$

where $f_{p}: \mathbb{R}^{n_{p}} \times \mathbb{R}^{n_{c}} \rightarrow \mathbb{R}^{n_{p}}$ and $f_{c}: \mathbb{R}^{n_{c}} \times \mathbb{R}^{n_{p}} \rightarrow \mathbb{R}^{n_{c}}$. We will assume that $\bar{f}_{p}, \bar{f}_{c}$ and $\bar{h}_{c}$ are such that $f_{p}$ and $f_{c}$ are continuous functions satisfying $f_{p}(0,0)=0$ and $f_{c}(0,0)=0$. 
The contribution of this paper is to design a suitable reset rule, or jump law, for controller (2), and to design a partition of the state space $\mathbb{R}^{n}$ (where $n=n_{p}+n_{c}$ ) in two subsets, called flow and jump sets. The state $x_{c}$ of controller (2) endowed with such additional logic, is then instantaneously reset according to the jump law whenever the state belongs to the jump set. This extended scheme, which is allowed to flow according to (3) only if the state belongs to the flow set, defines a hybrid system. More specifically, the proposed hybrid augmentation is designed to guarantee the decrease of one or both of two scalar Lyapunov-like functions, one of them $V: \mathbb{R}^{n_{p}} \times \mathbb{R}^{n_{c}} \rightarrow \mathbb{R}_{\geq 0}$, defined on the whole state space, and the other one $V_{p}: \mathbb{R}^{n_{p}} \rightarrow \mathbb{R}_{\geq 0}$ defined only in the plant state subspace. The functions $V$ and $V_{p}$ can be selected to capture some closed-loop stability and performance property (see the developments in Section 3.2 where output overshoot reduction is tackled). Functions $V$ and $V_{p}$ are linked to each other by a function $\phi: \mathbb{R}^{n_{p}} \rightarrow \mathbb{R}^{n_{c}}$ such that for all $\left(x_{p}, x_{c}\right)$ in $\mathbb{R}^{n_{p}} \times \mathbb{R}^{n_{c}}$

$$
V\left(x_{p}, \phi\left(x_{p}\right)\right) \leq V\left(x_{p}, x_{c}\right),
$$

and, in particular, by the relation

$$
V_{p}\left(x_{p}\right)=V\left(x_{p}, \phi\left(x_{p}\right)\right), \forall x_{p} \in \mathbb{R}^{n_{p}} .
$$

Within the above scenario, we will design flow and jump sets and jump rules such that the arising hybrid systems guarantee non-increase of $V$ or $V_{p}$, or both of them in two relevant cases:

$(V)$ (addressed in Section 2.1) where the function $V(\cdot, \cdot)$ is given and satisfies suitable conditions guaranteeing the existence ${ }^{2}$ of $\phi(\cdot)$ from which $V_{p}(\cdot)$ can be derived according to (5);

$\left(V_{p}\right)$ (addressed in Section 2.2) where $V_{p}(\cdot)$ and $\phi(\cdot)$ are given (their existence resembles an asymptotic controllability assumption), from which $V(\cdot, \cdot)$ satisfying (4) and (5) will be constructed.

Section 3 deals with the special case where system (3) is linear. In this case, quadratic versions of $V$ and $V_{p}$ can be constructed under reasonably weak properties required for the closed-loop dynamics. The extension to the linear case allows to strengthen the nonlinear results by exploiting the homogeneity property of hybrid systems acting on cones and obeying linear flow and jump rules. Finally, as a last contribution of this paper, we will show how to design $V_{p}$ in item $\left(V_{p}\right)$ to augment linear continuous-time control systems with hybrid loops that reduce the overshoot of a scalar plant output. In comparison to previous work, the aim of this paper is to design hybrid strategies to guarantee some asymptotic stability property by enforcing that suitable Lyapunov-like functions are not increasing along the hybrid solutions.

\footnotetext{
2 Here, to keep the discussion simple, it is assumed that $V$ is continuously differentiable and that there exists $\phi\left(x_{p}\right) \in$ $\operatorname{argmin} V\left(x_{p}, x_{c}\right)$, which implies (4). $x_{c} \in \mathbb{R}^{n_{c}}$
}

The arising hybrid closed loop resembles the so-called impulsive systems, considered e.g. in Haddad et al. (2001). However the objectives of Haddad et al. (2001) and of the present paper are different. Indeed an inverse optimal control involving a hybrid nonlinearnon-quadratic performance functional is developed in Haddad et al. (2001), whereas here we provide a design method of a hybrid loop (namely the jump map and the jump/flow sets) to ensure asymptotic stability and non-increase of suitable scalar functions. Our results are also linked to the event-triggered control literature (see Anta and Tabuada (2010)) for stability analysis of networked control systems, where it is necessary to reduce the number of times when the state is measured by the controller and the actuators are updated. The most important difference between the results mentioned above and our contribution is that in those works the resetting value for the state is uniquely associated to the transmission of a measurement sample, whereas in our results it depends on the Lyapunov-like functions that should not increase along solutions. Preliminary results in the direction of the work of this paper have been presented, without proofs, in Prieur et al. (2010, 2011). Our preliminary work also contains additional examples, not reported here due to space constraints. The present paper provides an improved discussion of the preliminary results, together with their proofs.

\section{Main results: nonlinear case}

\subsection{Constructing $V_{p}$ from $V$}

In this section we consider the closed-loop nonlinear system (3) and a function $V$ of the closed-loop state to address item $(V)$ of Section 1. To this aim, we make the following assumption on the function $V$.

Assumption 1 The function $V: \mathbb{R}^{n} \rightarrow \mathbb{R}_{\geq 0}$ is continuously differentiable and such that there exists a continuous differentiable function $\phi: \mathbb{R}^{n_{p}} \rightarrow \mathbb{R}^{n_{c}}$ such that

$$
\phi\left(x_{p}\right) \in \operatorname{argmin}_{x_{c} \in \mathbb{R}^{n_{c}}} V\left(x_{p}, x_{c}\right) .
$$

Moreover, there exists a class $\mathcal{K}$ function $\alpha$ such that, for all $x_{p}$ in $\mathbb{R}^{n_{p}}, x_{p} \neq 0$,

$$
\left\langle\nabla_{p} V\left(x_{p}, \phi\left(x_{p}\right)\right), f_{p}\left(x_{p}, \phi\left(x_{p}\right)\right)\right\rangle<-\alpha\left(V\left(x_{p}, \phi\left(x_{p}\right)\right)\right.
$$

where $\nabla_{p} V$ denotes the gradient of $V$ with respect to its first argument.

Remark 1 In Assumption 1 we do not impose that (3) is globally asymptotically stable, because (7) requires the function $V$ to be decreasing only in the subset of the state space defined by $\left(x_{p}, x_{c}\right)=\left(x_{p}, \phi\left(x_{p}\right)\right)$. Nevertheless, if system (3) is globally asymptotically stable, then there exist a function $V: \mathbb{R}^{n} \rightarrow \mathbb{R}_{\geq 0}$ and a class 
$\mathcal{K}$ function $\alpha$ such that $\langle\nabla V(x), f(x)\rangle<-\alpha(V(x))$ for all $x \neq 0$, which implies (7). Moreover note that in Assumption 1 , it is not required that $\operatorname{argmin}_{x_{c} \in \mathbb{R}} V\left(x_{p}, x_{c}\right)$ is a single valued map, but only that a continuous differentiable selection of this map does exist. For example, with $V\left(x_{p}, x_{c}\right)=x_{p}^{4}+x_{c}^{4}-x_{p}^{2} x_{c}^{2}$, we have $\operatorname{argmin}_{x_{c} \in \mathbb{R}} V\left(x_{p}, x_{c}\right)=\left\{x_{p},-x_{p}\right\}$ which is not a singleton even though Assumption 1 can be satisfied, e.g., with $\phi\left(x_{p}\right)=x_{p}$.

A natural way to stabilize the closed-loop system (3) is to flow when one (or both) of $V$ and $V_{p}$ is strictly decreasing and to reset the $x_{c}$-component of the state to the value $\phi\left(x_{p}\right)$ (where strict decrease is guaranteed by (7)) when the function is not decreasing. This leads to the following hybrid system ${ }^{3}$

$$
\begin{array}{rlrl}
\dot{x} & =f(x) & & \text { if } x \in \hat{F}, \\
\left(x_{p}^{+}, x_{c}^{+}\right) & =\left(x_{p}, \phi\left(x_{p}\right)\right) & \text { if } x \in \hat{J},
\end{array}
$$

where $\hat{F} \subset \mathbb{R}^{n}$ and $\hat{J} \subset \mathbb{R}^{n}$ are suitable closed subsets of the state space such that $\hat{F} \bigcup \hat{J}=\mathbb{R}^{n}$. In particular, $\hat{F}$ and $\hat{J}$ are defined by suitably combining the following two pairs of sets arising, respectively, from the knowledge of $V$ and $V_{p}$ :

$$
\begin{array}{r}
F=\left\{x \in \mathbb{R}^{n},\langle\nabla V(x), f(x)\rangle \leq-\bar{\alpha}(V(x))\right\} \\
J=\left\{x \in \mathbb{R}^{n},\langle\nabla V(x), f(x)\rangle \geq-\bar{\alpha}(V(x))\right\} \\
\bar{F}=\left\{x \in \mathbb{R}^{n},\left\langle\nabla V_{p}\left(x_{p}\right), f_{p}\left(x_{p}, x_{c}\right)\right\rangle \leq-\bar{\alpha}\left(V_{p}\left(x_{p}\right)\right)\right\} \\
\bar{J}=\left\{x \in \mathbb{R}^{n},\left\langle\nabla V_{p}\left(x_{p}\right), f_{p}\left(x_{p}, x_{c}\right)\right\rangle \geq-\bar{\alpha}\left(V_{p}\left(x_{p}\right)\right)\right\}
\end{array}
$$

where $\bar{\alpha}$ is any class $\mathcal{K}$ function such that $\bar{\alpha}(s) \leq \alpha(s)$ for all $s \geq 0$ (this will be denoted next by the shortcut notation $\bar{\alpha} \leq \alpha$ ). We state next our first main result whose proof is reported in Section 4.

Theorem 1 Consider the closed-loop system (3) and a function $V \in C^{1}$. Assume that there exist functions $\phi$ and $\alpha$ satisfying Assumption 1. Then for any class $\mathcal{K}$ function $\bar{\alpha}$ satisfying $\bar{\alpha} \leq \alpha$ the following holds.

1. If $V$ is positive definite and radially unbounded, then the hybrid system (8), (9) with $\hat{F}=F$ and $\hat{J}=$ $J$ is globally asymptotically stable and $V$ is nonincreasing along solutions.

2. If $V_{p}$, defined in (5), is positive definite and radially unbounded, then the hybrid system (8), (10) with $\hat{F}=\bar{F}$ and $\hat{J}=\bar{J}$ is such that the plant state $x_{p}$ converges to zero, and $V_{p}$ is non-increasing along solutions.

\footnotetext{
3 For an introduction of the hybrid systems framework used in this paper, see, e.g., the survey Goebel et al. (2009) or the brief overview in (Nešić et al., 2011, §2).
}

3. If $V$ is positive definite and radially unbounded, then the hybrid system (8), (9), (10) with $\hat{F}=F \bigcap \bar{F}$ and $\hat{J}=J \bigcup \bar{J}$ is globally asymptotically stable and both $V$ and $V_{p}$ are non-increasing along solutions.

Remark 2 The three items of Theorem 1 address, respectively, the three goals of guaranteeing suitable stability properties together with non-increase of $V, V_{p}$ and both of them. In particular, the flow and jump sets of the hybrid system (8) are defined in Theorem 1 from the knowledge of the Lyapunov-like function $V$. Note also that the hybrid system (8), (10) with $\hat{F}=\bar{F}$ and $\hat{J}=\bar{J}$ characterized at item 2 may be unstable. In other words, with this selection of the flow and jump sets, the plant state converges to the origin, although the state of the controller may diverge (which may complicate the implementation of the associate controller). In the linear case, this item will be strengthened under a detectability assumption.

\subsection{Constructing $V$ from $V_{p}$}

In this section we consider the closed-loop nonlinear system (3) and a function $V_{p}$ of the plant state to address item $\left(V_{p}\right)$ of Section 1 . To this aim, we make the following assumption on the function $V_{p}$.

Assumption 2 The function $V_{p}: \mathbb{R}^{n_{p}} \rightarrow \mathbb{R}_{\geq 0}$ is continuously differentiable and radially unbounded and there exist a continuously differentiable function $\phi: \mathbb{R}^{n_{p}} \rightarrow$ $\mathbb{R}^{n_{c}}$, and a class $\mathcal{K}$ function $\alpha$ such that, for all $x_{p}$ in $\mathbb{R}^{n_{p}}, x_{p} \neq 0$,

$$
\left\langle\nabla V_{p}\left(x_{p}\right), f_{p}\left(x_{p}, \phi\left(x_{p}\right)\right)\right\rangle<-\alpha\left(V_{p}\left(x_{p}\right)\right) .
$$

Note that, when the $x_{p}$-equation of the closed-loop system (3) is affine with respect to $x_{c}$, this condition is related to the asymptotic controllability to the origin (Artstein (1983)). In this case, a control law $\phi$ can be computed from a Control Lyapunov Function $V_{p}$ and from the so-called universal formulas (see Freeman and Kokotović (1996); Lin and Sontag (1991)).

Assumption 2 is sufficient to construct a function $V$ satisfying Assumption 1 so that the design strategy of the previous section can be employed. In particular, let $M$ be any positive semidefinite matrix ${ }^{4}$ in $\mathbb{R}^{n_{c}} \times \mathbb{R}^{n_{c}}$ and define $V: \mathbb{R}^{n_{p}} \times \mathbb{R}^{n_{c}} \rightarrow \mathbb{R}_{\geq 0}$ for all $\left(x_{p}, x_{c}\right)$ in $\mathbb{R}^{n_{p}} \times \mathbb{R}^{n_{c}}$,

$$
V(x)=V_{p}\left(x_{p}\right)+\left(x_{c}-\phi\left(x_{p}\right)\right)^{\prime} M\left(x_{c}-\phi\left(x_{p}\right)\right) .
$$

Note that $V$ is continuously differentiable, and radially unbounded. Moreover, if $\phi(0)=0$ and $M>0$, then

\footnotetext{
${ }^{4}$ Note that the matrix $M$ may be a function of $x$. This extra degree of freedom could be used to perform convenient selections of $V$.
} 
it is a positive definite function because, for each $x_{p}$, it is the sum of two positive definite terms, the first one strictly positive when $x_{p} \neq 0$ and the second one strictly positive when $x_{p}=0$. The following theorem is a straightforward application of Theorem 1 in light of the $V_{p}$ and $\phi$ given in Assumption 2 and of the $V$ in (12). Its proof is reported in Section 4. An nonlinear example illustrating the potential behind the nonlinear design of Theorem 1 has been reported in Prieur et al. (2010) and is not reported here due to space constraints.

Theorem 2 Consider the closed-loop system (3) and a function $V_{p} \in C^{1}$. Assume that there exist functions $\phi$ and $\alpha$ satisfying Assumption 2. Given any positive semidefinite matrix $M \in \mathbb{R}^{n_{c}} \times \mathbb{R}^{n_{c}}$, the corresponding function $V$ in (12), and any class $\mathcal{K}$ function $\bar{\alpha}$ satisfying $\bar{\alpha} \leq \alpha$, the three items of Theorem 1 hold.

\section{Main results: linear case}

\subsection{Corollaries and extension of Theorems 1 and 2}

When focusing on linear dynamics, the two items $(V)$ and $\left(V_{p}\right)$ in Section 1 are addressed here. Consider the following linear plant:

$$
\dot{x}_{p}=\bar{A}_{p} x_{p}+\bar{B}_{p} u,
$$

with $x_{p} \in \mathbb{R}^{n_{p}}$, in feedback interconnection with a (not necessarily stabilizing) linear dynamic controller:

$$
\dot{x}_{c}=\bar{A}_{c} x_{c}+\bar{B}_{c} x_{p}, \quad u=\bar{C}_{c} x_{c}+\bar{D}_{c} x_{p},
$$

with $x_{c} \in \mathbb{R}^{n_{c}}$. The closed loop is described by the following linear system

$$
\dot{x}=A x:=\left[\begin{array}{ll}
A_{p} & B_{p} \\
B_{c} & A_{c}
\end{array}\right] x,
$$

where $x=\left(x_{p}^{\prime}, x_{c}^{\prime}\right)^{\prime}$ and $A_{p}, B_{p}, A_{c}$, and $B_{c}$ are matrices of appropriate dimensions uniquely based on the matrices in (13) and (14). In the linear case, it is reasonable to restrict $V$ and $V_{p}$ to the class of quadratic functions, $\phi$ to the class of linear stabilizers and $\alpha$ and $\bar{\alpha}$ to the class of linear gains. Based on this, the closedloop function $V$ can be selected as $V(x)=x^{\prime} P x$ where $P=\left[\begin{array}{cc}P_{p} & P_{p c} \\ P_{p c}^{\prime} & P_{c}\end{array}\right]$ is a symmetric positive definite matrix. Then, since $\nabla_{c} V\left(x_{p}, x_{c}\right)=2\left(P_{p c}^{\prime} x_{p}+P_{c} x_{c}\right)$, from the positive definiteness of $P$, the critical points of $V$ are the points where $V$ is minimal. Thus the unique function $\phi: \mathbb{R}^{n_{p}} \rightarrow \mathbb{R}^{n_{c}}$ satisfying (4) is given by, for all $x_{p} \in \mathbb{R}^{n_{p}}$,

$$
\phi\left(x_{p}\right)=-P_{c}^{-1} P_{p c}^{\prime} x_{p}=K_{p} x_{p} .
$$

Moreover, the function $V_{p}: \mathbb{R}^{n_{p}} \rightarrow \mathbb{R}_{\geq 0}$ defined by (5) becomes, for all $x_{p} \in \mathbb{R}^{n_{p}}$,

$$
\begin{aligned}
V_{p}\left(x_{p}\right) & =\left[\begin{array}{c}
x_{p} \\
-P_{c}^{-1} P_{p c}^{\prime} x_{p}
\end{array}\right]^{\prime}\left[\begin{array}{cc}
P_{p} & P_{p c} \\
\star & P_{c}
\end{array}\right]\left[\begin{array}{c}
x_{p} \\
-P_{c}^{-1} P_{p c}^{\prime} x_{p}
\end{array}\right] \\
& =x_{p}^{\prime}\left(P_{p}-P_{p c} P_{c}^{-1} P_{p c}^{\prime}\right) x_{p}=x_{p}^{\prime} \bar{P}_{p} x_{p} .
\end{aligned}
$$

Based on (16) and (17), we get that (7) holds as long as there exists $\widetilde{\alpha}>0$ such that

$$
\operatorname{He}\left(\bar{P}_{p}\left(A_{p}+B_{p} K_{p}\right)\right)<-\widetilde{\alpha} \bar{P}_{p} .
$$

Thus, given $V$, if such a $\widetilde{\alpha}>0$ exists, then Assumption 1 is guaranteed. Consider now the sets in (9) and (10). Given any $0<\overline{\widetilde{\alpha}} \leq \widetilde{\alpha}$ and with the definitions above, after some calculations they become

$$
\begin{gathered}
F=\left\{x \in \mathbb{R}^{n}, x^{\prime} N x \leq-\overline{\widetilde{\alpha}} x^{\prime} P x\right\}, \\
J=\left\{x \in \mathbb{R}^{n}, x^{\prime} N x \geq-\overline{\widetilde{\alpha}} x^{\prime} P x\right\}, \\
\bar{F}=\left\{x \in \mathbb{R}^{n}, x^{\prime} N_{p} x \leq-\overline{\widetilde{\alpha}} x_{p}^{\prime} \bar{P}_{p} x_{p}\right\}, \\
\bar{J}=\left\{x \in \mathbb{R}^{n}, x^{\prime} N_{p} x \geq-\overline{\widetilde{\alpha}} x_{p}^{\prime} \bar{P}_{p} x_{p}\right\},
\end{gathered}
$$

where $N=\operatorname{He}\left[\begin{array}{ccc}P_{p} A_{p}+P_{p c} B_{c} & P_{p c} A_{c}+P_{p} B_{p} \\ P_{p c}^{\prime} A_{p}+P_{c} B_{c} & P_{c} A_{c}+P_{p c}^{\prime} B_{p}\end{array}\right]$ and $N_{p}=$ He $\left[\begin{array}{cc}\bar{P}_{p} A_{p} & \bar{P}_{p} B_{p} \\ 0 & 0\end{array}\right]$. With the above definitions, the following corollary particularizes the results of Theorem 1 to the linear case.

Corollary 1 Consider the closed-loop system (15) and a function $V(x)=x^{\prime} P x=x^{\prime}\left[\begin{array}{cc}P_{p} & P_{p c} \\ P_{p c}^{\prime} & P_{c}\end{array}\right] x$ such that $\bar{P}_{p}=$ $P_{p}-P_{p c} P_{c}^{-1} P_{p c}^{\prime}$ satisfies (18) for some $\widetilde{\alpha}>0$ and for $K_{p}=-P_{c}^{-1} P_{p c}^{\prime}$. Then the hybrid system

$$
\begin{array}{rlrl}
\dot{x} & =A x & & \text { if } x \in \hat{F}, \\
\left(x_{p}, x_{c}\right)^{+} & =\left(x_{p}, K_{p} x_{p}\right) & \text { if } x \in \hat{J},
\end{array}
$$

satisfies all the items of Theorem 1 with $V_{p}\left(x_{p}\right)=x_{p}^{\prime} \bar{P}_{p} x_{p}$ and using the sets in (19) with any $0<\overline{\widetilde{\alpha}} \leq \widetilde{\alpha}$.

Remark 3 Let us apply Corollary 1 to linear plants in closed loop with reset controllers (see e.g. Beker et al. (2004); Nešić et al. (2008)). Consider the linear plant (13) with $\bar{A}_{p}$ Hurwitz, $\bar{C}_{c}=I_{n_{c}}$ and $\bar{D}_{c}=0$. Recalling the notation in (15), when closing the loop with (14), we get $A_{p}=\bar{A}_{p}$ and $B_{p}=\bar{B}_{p}$, and thus $A_{p}$ is Hurwitz. To represent reset controllers, let us consider $K_{p}=0$. Then there exist a symmetric positive definite matrix $\bar{P}_{p}$ in $\mathbb{R}^{n_{p} \times n_{p}}$ and $\widetilde{\alpha}>0$ such that (18) holds. Applying Corollary 1 gives that for each symmetric positive definite matrix $P_{c}$ in $\mathbb{R}^{n_{c} \times n_{c}}$, the hybrid system

$$
\begin{aligned}
\dot{x} & =A x \quad \text { if } x \in F_{l}, \\
\left(x_{p}, x_{c}\right)^{+} & =\left(x_{p}, 0\right) \text { if } x \in J_{l},
\end{aligned}
$$

where the flow and the jump sets are defined with $N_{l}=$ $\mathrm{He}\left[\begin{array}{lll}P_{p} A_{p} & P_{p} B_{p} \\ P_{c} B_{c} & P_{c} A_{c}\end{array}\right]$, and for any $\overline{\widetilde{\alpha}} \leq \widetilde{\alpha}$, by

$$
\begin{aligned}
& F_{l}=\left\{x \in \mathbb{R}^{n}, x^{\prime} N_{l} x \leq-\overline{\widetilde{\alpha}} x^{\prime} P x\right\} \\
& J_{l}=\left\{x \in \mathbb{R}^{n}, x^{\prime} N_{l} x \geq-\overline{\widetilde{\alpha}} x^{\prime} P x\right\},
\end{aligned}
$$

is globally asymptotically stable and the function $V_{l}$ defined by $V_{l}(x)=x^{\prime} P x$, for any $x$ in $\mathbb{R}^{n}$, and for $P=\operatorname{diag}\left(P_{p}, P_{c}\right)$, is non-increasing. 
Let us now specialize Theorem 2 to the linear case. With (15), Assumption 2 is satisfied whenever the pair $\left(A_{p}, B_{p}\right)$ is stabilizable and $V_{p}\left(x_{p}\right)=x_{p}^{\prime} \bar{P}_{p} x_{p}$, with $\bar{P}_{p}>$ 0 , is a control Lyapunov function for $\left(A_{p}, B_{p}\right)$. As a matter of fact in that case there exist a static state feedback matrix $K_{p}$ and a constant $\widetilde{\alpha}>0$ such that equation (18) holds, and then equation (11) will hold with $\phi\left(x_{p}\right)=K_{p} x_{p}$. In particular, given $\vec{P}_{p}, K_{p}$ and $\widetilde{\alpha}$ can be computed using a Linear Matrix Inequality (LMI) solver. Alternatively, under a stabilizability assumption, one can always solve a generalized eigenvalue problem (GEVP) and find an optimal pair $\left(\bar{P}_{p}, K_{p}\right)$ maximizing $\widetilde{\alpha}$. Based on $V_{p}$ and $K_{p}$, consider any symmetric positive definite matrix $P_{c}$ in $\mathbb{R}^{n_{c} \times n_{c}}$. The function $V: \mathbb{R}^{n_{p}} \times \mathbb{R}^{n_{c}} \rightarrow \mathbb{R}_{\geq 0}$ in (12) can be defined as

$$
\begin{aligned}
V(x) & =V_{p}\left(x_{p}\right)+\left(x_{c}-K_{p} x_{p}\right)^{\prime} P_{c}\left(x_{c}-K_{p} x_{p}\right) \\
& =x^{\prime}\left[\begin{array}{cc}
\bar{P}_{p}+K_{p}^{\prime} P_{c} K_{p} & -K_{p}^{\prime} P_{c} \\
-P_{c} K_{p} & P_{c}
\end{array}\right] x=x^{\prime} P x .
\end{aligned}
$$

Since $P_{c}>0$, this function is continuously differentiable, radially unbounded and positive definite. Then the following corollary of Theorem 2 can be stated.

Corollary 2 Assume that the pair $\left(A_{p}, B_{p}\right)$ is stabilizable and that $V_{p}(x)=x_{p}^{\prime} \bar{P}_{p} x_{p}$ is a control Lyapunov function for this pair. Then there exist $\widetilde{\alpha}>0$ and $K_{p}$ satisfying (18). Moreover, given any symmetric positive definite matrix $P_{c}$ in $\mathbb{R}^{n_{c} \times n_{c}}$ and the corresponding function $V$ defined in (22), for any selection of $0<\widetilde{\widetilde{\alpha}} \leq \widetilde{\alpha}$, the reset system (20) with the sets in (19) satisfies all the items of Theorem 1.

Remark 4 The global asymptotic stability (GAS) results established at items 1 and 3 of Theorem 1 can be strengthened to global exponential stability (GES) in the case of Corollaries 1 and 2. Indeed, the reset system (19), (20) corresponds to a linear dynamics acting on conic flow and jump sets and, (Nešić et al., 2011, Theorem 7) can be applied to conclude that GAS implies GES. See also the proof of Theorem 3 in Section 4 for a similar reasoning.

As already pointed out in Remark 2, when selecting $\hat{F}=\bar{F}$ and $\hat{J}=\bar{J}$, as in item 2 of Theorem 1 , there is no guarantee that the state of the controller will converge to zero. This property is instead guaranteed when using $\hat{F}=\bar{F} \bigcap F$ and $\hat{J}=\bar{J} \bigcup J$, as in item 3 of Theorem 1 . However, in light of Corollary 2, it would be desirable to provide conditions under which using the sets at item 2 of Theorem 1 is already sufficient to guarantee that the controller states converge to zero too. The advantage of this is that one would not need to bother with the selection of the matrix $P_{c}$ of Corollary 2 , because the sets $\bar{F}$ and $\bar{J}$ only depend on $V_{p}$ and $K_{p}$. In the linear case, it turns out to be sufficient that the controller dynamics is detectable from the output matrix $B_{p}$. Intuitively, this requirement corresponds to asking that any nonzero controller evolution will be detected by the plant states so that a LaSalle result can be applied to show convergence. The result is formalized in the next theorem whose proof is reported in Section 4.

Theorem 3 Consider any gain $K_{p}$ such that $A_{p}+B_{p} K_{p}$ is Hurwitz and a pair $\bar{P}_{p}, \widetilde{\alpha}$ satisfying (18). Consider any $\overline{\widetilde{\alpha}} \leq \widetilde{\alpha}$ and the flow and jump sets in (19b). If the pair $\left(B_{p}, A_{c}\right)$ is detectable, then the hybrid system at item 2 of Theorem 1 is globally exponentially stable.

\subsection{Performance-based designs}

In this section, we rely on the results of Theorem 3 to propose a suitable jump rule to be incorporated in a linear continuous-time control system to reduce the plant output overshoot. See also the design of a jump rule to maximize the decay rate in Prieur et al. (2011).

In Beker et al. (2001) a reset controller was shown to improve upon linear control in terms of overshoot reduction. If the plant (13) is strictly proper from a certain output $y$, namely $y=\bar{C}_{p} x_{p}$, a possible way to mathematically formalize the requirement of overshoot reduction in terms of the Lyapunov functions introduced here is to construct a quadratic function $V_{p}\left(x_{p}\right):=x_{p}^{\prime} \bar{P}_{p} x_{p}$ and the stabilizing gain $K_{p}$ in such a way that, to a certain extent, $V_{p}\left(x_{p}\right) \approx|y|^{2}$, where $y$ is a given scalar output. Then achieving non-increase of $V_{p}$ via the hybrid loops of this paper will induce (almost) no overshoot, namely $|y(t)|$ is (almost) non-increasing along trajectories. Finding the above discussed pair $\left(V_{p}, K_{p}\right)$ can be done, for example, by writing the dynamics (13) in observability canonical form, so that $x_{p}=\left[\begin{array}{c}x_{1} \\ y\end{array}\right]$ and solving the following LMI eigenvalue problem:

$$
\begin{aligned}
\min _{\bar{Q}_{p}}={\overline{\bar{Q}_{p}^{\prime}}}_{,}^{\prime}, \rho_{x}, \rho_{y}, X & \rho_{y}, \text { s.t. } \quad \bar{Q}_{p}=\left[\begin{array}{cc}
Q_{1} & q_{1 y} \\
q_{1 y}^{\prime} & q_{y}
\end{array}\right]>I \\
0 & <\left[\begin{array}{cc}
\kappa_{M} I & X \\
X^{\prime} & \kappa_{M} I
\end{array}\right], \quad 0>\operatorname{He}\left(A_{p} \bar{Q}_{p}+B_{p} X\right) \\
0 & \leq\left[\begin{array}{cc}
\rho_{x} & 1 \\
1 & \rho_{y}
\end{array}\right], \quad \rho_{x}<Q_{1}, \quad q_{y}<1+\rho_{y},
\end{aligned}
$$

where $\kappa_{M}>0$ is given. The optimal solution to (23) will lead to the gain $K_{p}=X \bar{Q}_{p}^{-1}$ and to $\bar{P}_{p}=\bar{Q}_{p}^{-1}$ satisfying (18) with a small enough $\widetilde{\alpha}$ and such that $\left|K_{p}\right| \leq$ $|X|\left|\bar{Q}_{p}^{-1}\right| \leq \kappa_{M}$. Moreover, the bounds given by the last three constraints can be shown to imply that smaller values of $\rho_{y}$ will lead to a function $V_{p}\left(x_{p}\right)=x_{p}^{\prime} \bar{Q}_{p}^{-1} x_{p}$ closer to $|y|^{2}$. This fact, together with desirable stability properties of the hybrid closed loop, is established in the following proposition whose proof is given in Section 4.

Proposition 1 Consider a sequence of solutions $\left(\bar{Q}_{p}^{k}\right.$, $\left.\rho_{y}^{k}, \rho_{x}^{k}, X^{k}\right)_{k \in \mathbb{N}}$ to the optimization (23) such that $\rho_{y}^{k} \rightarrow 0$ 
as $k \rightarrow \infty$. Then, defining $V_{p}^{k}\left(x_{p}\right)=x_{p}^{\prime}\left(\bar{Q}_{p}^{k}\right)^{-1} x_{p}$, we have for each $x_{p}, \lim _{k \rightarrow \infty} V_{p}^{k}\left(x_{p}\right)=|y|^{2}$, namely as $\rho_{y}^{k}$ approaches zero, $V_{p}^{k}\left(x_{p}\right)$ approaches $|y|^{2}$.

Moreover, given any pair $\left(\bar{Q}_{p}, X\right)$ satisfying (23), let $K_{p}=X \bar{Q}_{p}^{-1}$ and $\bar{P}_{p}=\bar{Q}_{p}^{-1}$. Then for a small enough selection of $\widetilde{\alpha}$, equation (18) is satisfied. Then, given any selection of $0<\overline{\widetilde{\alpha}} \leq \widetilde{\alpha}$, the reset system (20) with the flow and jump sets in (19b), is such that:

1. the plant state $x_{p}$ converges to zero, and the function $V_{p}\left(x_{p}\right)$ is non-increasing along solutions;

2. if the pair $\left(B_{p}, A_{c}\right)$ is detectable, then the hybrid closed loop is globally exponentially stable.

From the point of view of the overshoot reduction, the meaning of the first statement of Proposition 1 is that smaller values of $\rho_{y}$ (which are expected to be achievable as the bound $\kappa_{M}$ on $\left|K_{p}\right|$ is increased) will guarantee that the function $V_{p}\left(x_{p}\right)$ becomes closer to the squared value $|y|^{2}$ of the output. One way to address overshoot elimination is to guarantee that the function $|y|^{2}$ itself is not increasing along solutions (indeed, as soon as $y(t)=0$ for some $t$, then $y$ will remain zero). Since our hybrid scheme ensures that $V_{p}$ does not increase along solutions, then the design in (23) leads to an "almost" non overshooting closed loop as long as $\rho_{y}$ is small enough. On the other hand, note that $\rho_{y}=0$ (namely, total overshoot elimination) cannot be achieved because we require that $V_{p}$ be positive definite in our theory.

Example 1 We consider an example originally discussed in Beker et al. (2004), involving a First Order Reset Element (FORE), whose flow dynamics follow the continuous-time transfer function $\frac{1}{s+1}$ and whose jump rule is to reset the state to zero whenever the input and the output of the FORE have opposite signs. This FORE is interconnected in negative unit feedback with a SISO plant whose transfer function is $\mathcal{P}=\frac{s+1}{s(s+0.2)}$. For this example, the control system involving the FORE is shown in Beker et al. (2004) to behave more desirably than the linear control system because it has only about $40 \%$ overshoot as compared to the linear closed-loop system, while retaining the rise time of the linear design (this example was also discussed later in Nešić et al. (2005); Zaccarian et al. (2011) where the $\mathcal{L}_{2}$ gain properties of the reset closed loop were characterized). Here we show that when allowing for more general resets than just the ones induced by the FORE, arbitrary small overshoot can be achieved, while retaining the same rise time. To this aim, we use the following state-space representation of the linear closed-loop system: $\left[\begin{array}{l|l}A_{p} & B_{p} \\ \hline B_{c} & A_{c}\end{array}\right]=\left[\begin{array}{cc|c|c}-0.6 & 0.6 & -1 \\ \hline-0.4 & 0.4 & 1 \\ \hline 0 & 1 & -1\end{array}\right]$. Table 1 reports the values of $\bar{P}_{p}, K_{p}$ and $\rho_{y}^{-1}$ for some selections of $\kappa_{M}$. Using the values in Table 1 , we run several simulations

\begin{tabular}{|c|c|c|c|}
\hline$\kappa_{M}$ & $\bar{P}_{p}$ & $K_{p}^{\prime}$ & $\rho_{y}^{-1}$ \\
\hline 0.1 & {$\left[\begin{array}{cc}0.31315 & -0.38064 \\
-0.38064 & 0.78906\end{array}\right]$} & {$\left[\begin{array}{c}0.046750 \\
-0.087302\end{array}\right]$} & 0.48451 \\
0.5 & {$\left[\begin{array}{cc}0.10170 & -0.088509 \\
-0.088509 & 0.99128\end{array}\right]$} & {$\left[\begin{array}{c}0.055896 \\
-0.49023\end{array}\right]$} & 10.662 \\
1 & {$\left[\begin{array}{cc}0.049336 & -0.045986 \\
-0.045986 & 0.99778\end{array}\right]$} & {$\left[\begin{array}{c}0.057423 \\
-0.97424\end{array}\right]$} & 21.179 \\
5 & {$\left[\begin{array}{cc}0.0097785 & -0.0096376 \\
-0.0096376 & 0.99991\end{array}\right]$} & {$\left[\begin{array}{l}0.059499 \\
-4.8307\end{array}\right]$} & 103.25 \\
\hline
\end{tabular}

Table 1

Matrices $\bar{P}_{p}, K_{p}$ and $\rho_{y}^{-1}$ for some selections of $\kappa_{M}$.

of the closed loop starting from the initial conditions $x_{p}(0,0)=\left[\begin{array}{ll}-1 & -1\end{array}\right]^{\prime}$, resembling the step responses reported in Beker et al. (2004); Nešić et al. (2005); Zaccarian et al. (2011). In particular, we implement the hybrid loops arising when using the values in the table within the scheme proposed in Theorem 1 . Note that since in this case the pair $\left(B_{p}, A_{c}\right)$ is observable, then according to the results of Theorem 3 , the trajectories are all convergent to zero. The resulting plant input and output responses are shown in Figure 1, where we also show the linear response (thin solid) and the response obtained with the FORE used in Beker et al. (2004); Nešić et al. (2005); Zaccarian et al. (2011) (thin dashed).
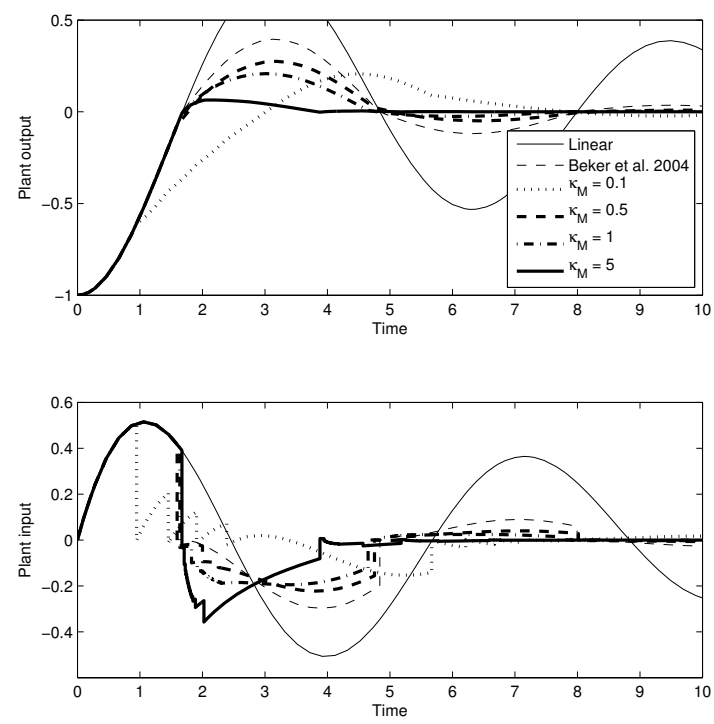

Fig. 1. Example 1: simulation results.

The results in Figure 1 are indicative of the potential of the proposed approach. It should be pointed out that although we manage to improve the overshoot reduction as compared to the FORE resetting strategy proposed in Beker et al. (2004) (thin dashed curve), this last strategy is more appealing from an implementation viewpoint because it only requires a measurement of the plant output. Conversely, the resetting strategy of the improved bold curves of the figure are obtained using a full measurement of the plant state. 


\section{Proofs of the technical results}

In Assumption 1 we introduce a continuously differentiable function $\phi: \mathbb{R}^{n_{p}} \rightarrow \mathbb{R}^{n_{c}}$ satisfying (6). However, for the results of this paper to hold, it is sufficient that $\phi$ is a Hölder continuous function of order strictly larger than $\frac{1}{2}$ satisfying (4). This regularity assumption on $\phi$ is weaker than the one required in Assumption 1. The following lemma (see also (Pan et al., 2001, Lemma 1)) provides the essential technical properties of the function $V_{p}$ (introduced in (5)) and the connection between the gradient ${ }^{5}$ of $V_{p}$ and that one of the function $V$ (introduced in (4)) required to carry out the proofs in the rest of the section.

Lemma 1 Assume that there exists a function $\phi$ : $\mathbb{R}^{n_{p}} \rightarrow \operatorname{argmin}_{z} V(x, z) \subset \mathbb{R}^{n_{c}}$ which is locally Hölder continuous of order strictly larger than $\frac{1}{2}$ satisfying Assumption 1. Then, there exists $M^{2}$ : $\mathbb{R}^{n_{p}} \times \mathbb{R}^{n_{c}} \rightarrow \mathbb{R}^{n_{c}} \times \mathbb{R}^{n_{c}}$ such that, for all $x=\left(x_{p}, x_{c}\right)$ in $\mathbb{R}^{n_{p}} \times \mathbb{R}^{n_{c}}$,

$$
\begin{aligned}
& \nabla V_{p}\left(x_{p}\right)=\nabla_{p} V(x, \phi(x)), \\
& V\left(x_{p}, x_{c}\right)=V_{p}\left(x_{p}\right) \\
&+\left(x_{c}-\phi\left(x_{p}\right)\right)^{\prime} M\left(x_{p}, x_{c}\right)\left(x_{c}-\phi\left(x_{p}\right)\right),
\end{aligned}
$$$$
\langle\nabla V(g(x)), f(g(x))\rangle=\left\langle\nabla V_{p}\left(x_{p}\right), f_{p}\left(x_{p}, \phi\left(x_{p}\right)\right)\right\rangle \text {. }
$$

Proof. Let us prove the equations of Lemma 1 successively. First note that (6) implies

$$
\nabla_{c} V\left(x_{p}, \phi\left(x_{p}\right)\right)=0 \text {. }
$$

Proof of (24). To simplify notation, we will use next, $V_{p h}\left(x_{p}, y_{p}\right):=V_{p}\left(x_{p}+h y_{p}\right)-V_{p}\left(x_{p}\right)$ and $\phi_{h}\left(x_{p}, y_{p}\right):=$ $\phi\left(x_{p}+h y_{p}\right)-\phi\left(x_{p}\right)$. Since $\phi$ is Hölder continuous of order strictly larger than $\frac{1}{2}$, there exists a positive real number $\varepsilon$ such that, for each $x_{p}$ in $\mathbb{R}^{n_{p}}$, we can find positive values $c$ and $h_{0}$ satisfying $\frac{\left|\phi_{h}\left(x_{p}, y_{p}\right)\right|}{\sqrt{|h|}}=\frac{\left|\phi\left(x_{p}+h y_{p}\right)-\phi\left(x_{p}\right)\right|}{\sqrt{|h|}} \leq$ $c|h|^{\varepsilon}$, for all $|h| \leq h_{0}$, and for all $y_{p} \in \mathbb{R}^{n_{p}}$ such that $\left|y_{p}\right|=1$. By using (27), we get, for each $y_{p}$ in $\mathbb{R}^{n_{p}}$ and $h>0$,

$$
\begin{aligned}
& \frac{V_{p h}\left(x_{p}, y_{p}\right)}{h}=\frac{V_{p}\left(x_{p}+h y_{p}\right)-V_{p}\left(x_{p}\right)}{h} \\
& =\int_{0}^{1} \nabla_{p} V\left(x_{p}+s h y_{p}, \phi\left(x_{p}+h y_{p}\right)\right) d s y_{p} \\
& \quad+\int_{0}^{1} \nabla_{c} V\left(x_{p}, \phi\left(x_{p}\right)+s \phi_{h}\left(x_{p}, y_{p}\right)\right) d s\left(\frac{\phi_{h}\left(x_{p}, y_{p}\right)}{h}\right) .
\end{aligned}
$$

But, with (27), we get:

$$
\begin{aligned}
& \nabla_{c} V\left(x_{p}, \phi\left(x_{p}\right)+s \phi_{h}\left(x_{p}, y_{p}\right)\right) \\
& =s \phi_{h}\left(x_{p}, y_{p}\right)^{\prime}\left(\int_{0}^{1} \frac{\partial^{2} V}{\partial x_{c}^{2}}\left(x_{p}, \phi\left(x_{p}\right)+s t \phi_{h}\left(x_{p}, y_{p}\right)\right) d t\right) .
\end{aligned}
$$

\footnotetext{
${ }^{5}$ In this section, $\nabla_{c} V$ denotes the gradient of $V$ along the $x_{c}$-direction.
}

This yields:

$$
\begin{array}{r}
\frac{V_{p h}\left(x_{p}, y_{p}\right)}{h}=\left(\int_{0}^{1} \nabla_{p} V\left(x_{p}+s h y_{p}, \phi\left(x_{p}+h y_{p}\right)\right) d s\right) y_{p} \\
+\frac{\phi_{h}\left(x_{p}, y_{p}\right)^{\prime}}{\sqrt{h}} \int_{0}^{1} s \int_{0}^{1} \frac{\partial^{2} V}{\partial x_{c}^{2}}\left(x_{p}, \phi\left(x_{p}\right)+s t \phi_{h}\left(x_{p}, y_{p}\right)\right) d t d s \\
\left(\frac{\phi_{h}\left(x_{p}, y_{p}\right)}{\sqrt{h}}\right),(28)
\end{array}
$$

which, taking the limit as $h$ goes to 0 , yields (24).

Proof of (25). Moreover, with (27), we get (25), with the notation $M\left(x_{p}, x_{c}\right)=\int_{0}^{1} \int_{0}^{1} \frac{\partial^{2} V}{\partial x_{c}^{2}}\left(x_{p}, \phi\left(x_{p}\right)+t s\left[x_{c}-\right.\right.$ $\left.\left.\phi\left(x_{p}\right)\right]\right) s d t d s$.

Proof of (26). Using (27), we get that for all $x$ in $\mathbb{R}^{n}$,

$$
\begin{aligned}
& \langle\nabla V(g(x)), f(g(x))\rangle \\
& =\left\langle\nabla_{p} V(g(x)), f_{p}(g(x))\right\rangle+\left\langle\nabla_{c} V(g(x)), f_{c}(g(x))\right\rangle \\
& =\left\langle\nabla_{p} V(g(x)), f_{p}(g(x))\right\rangle \\
& =\left\langle\nabla_{p} V\left(x_{p}, \phi\left(x_{p}\right)\right), f_{p}\left(x_{p}, \phi\left(x_{p}\right)\right)\right\rangle
\end{aligned}
$$

Moreover, equation (24) gives, for all $x_{p}$ in $\mathbb{R}^{n_{p}}$,

$$
\begin{aligned}
& \left\langle\nabla V_{p}\left(x_{p}\right), f_{p}\left(x_{p}, \phi\left(x_{p}\right)\right)\right\rangle \\
& \quad=\left\langle\nabla_{p} V\left(x_{p}, \phi\left(x_{p}\right)\right), f_{p}\left(x_{p}, \phi\left(x_{p}\right)\right)\right\rangle .
\end{aligned}
$$

Combining (29) and (30), we get (26).

The following lemma follows directly from (25) and (26) of Lemma 1 and from Assumption 1. Its proof is omitted.

Lemma 2 Given a solution of (8), (9) with $\hat{F}=F$ and $\hat{J}=J$ (respectively of (8), (10) with $\hat{F}=\bar{F}$ and $\hat{J}=\bar{J}$ ) which jumps at some time, after the jump the solution is either equal to zero (i.e. $g(x)=0$ ) or it has to flow.

Proof of Theorem 1. Item 1. Due to (9), when flowing, the function $V$ does not increase, and due to Lemma 2, after each jump, a solution of (8), (9) with $\hat{F}=F$ and $\hat{J}=J$ is equal to zero (i.e. $g(x)=0$ ), or has to flow, and thus, by definition of $F$, the function $V$ does not increase. This implies that the hybrid system (8), (9) with $\hat{F}=F$ and $\hat{J}=J$ is globally stable, and the function $V$ does not increase. To prove the attractivity of the system (8), (9) with $\hat{F}=F$ and $\hat{J}=J$, let us apply the LaSalle invariance property for hybrid systems. Let us consider a solution of (8), (9) with $\hat{F}=F$ and $\hat{J}=J$, which is included in a level set of the function $V$. Let us show that this solution should be equal to 0. Due to Lemma 2 , the solution either cannot jump (since after a jump, the solution has to flow, and thus the value of $V$ has to decrease), or has to be at the origin. Given a solution flowing for all time, it is a solution of the system (3) in a 
level set of the function $V$. Since $\bar{\alpha}$ is a function of class $\mathcal{K}$, then, due to $(9)$, the solution can not flow for all time and stay in a level set of $V$. Then the solution has to be constant and equal to the origin. Therefore, by (Goebel et al., 2009, Theorem 23, page 64), the system (8), (9) with $\hat{F}=F$ and $\hat{J}=J$ is globally asymptotically stable.

Item 2. Due to (10), when flowing, the function $V_{p}$ does not increase along the solutions of (8), (10) with $\hat{F}=\bar{F}$ and $\hat{J}=\bar{J}$. Moreover, due to Lemma 2, after each jump, the solution is equal to zero (i.e. $g(x)=0$ ), or has to flow, and thus, with (7), the function $V_{p}$ does not increase. This implies that the variable $x_{p}$ converges to zero. Since after a jump, the solutions of (8), (10) with $\hat{F}=\bar{F}$ and $\hat{J}=\bar{J}$ has to flow, it implies that, for each solution $x$, for all $(t, j)$ in $\operatorname{dom} x$, we have $\frac{d}{d t} V_{p}(x(t, j))<$ $-\bar{\alpha}\left(V_{p}\left(x_{p}(t, j)\right)\right)$ except when a jump occurs. Since during the jumps, the $V_{p}$ remains unchanged, and since the number of jumps is at most countable, we get that $V_{p}$ converges to 0 when $t \rightarrow \infty$, and thus $x_{p}$ converges to 0 when $t \rightarrow \infty$.

Item 3. This item follows from the previous two items.

Proof of Theorem 2. From (12), the gradient of $V$ is given for all $\left(x_{p}, x_{c}\right) \in \mathbb{R}^{n_{p}} \times \mathbb{R}^{n_{c}}$ by

$$
\begin{aligned}
\nabla_{p} V\left(x_{p}, x_{c}\right) & =\frac{\partial V_{p}}{\partial x_{p}}\left(x_{p}\right)-2\left(x_{c}-\phi\left(x_{p}\right)\right)^{\prime} M \frac{\partial \phi}{\partial x_{p}}\left(x_{p}\right) \\
\nabla_{c} V\left(x_{p}, x_{c}\right) & =2\left(x_{c}-\phi\left(x_{p}\right)\right)^{\prime} M
\end{aligned}
$$

Therefore, $\nabla_{c} V\left(x_{c}\right)=0$ whenever $x_{c}=\phi\left(x_{p}\right)$, which, by the positive semi-definiteness of $M$ implies that equation (6) holds. Moreover, by (26) of Lemma 1, it follows that (11) coincides with (7) and Assumption 1 holds. Thus Theorem 1 applies and this concludes the proof of Theorem 2.

Proof of Theorem 3. Consider any gain $K_{p}$ such that $A_{p}+B_{p} K_{p}$ is Hurwitz and a pair $\bar{P}_{p}, \alpha$ satisfying (18). Consider any symmetric positive definite matrix $P_{c}$ in $\mathbb{R}^{n_{c} \times n_{c}}$, and let $P_{p c}=-K_{p}^{\prime} P_{c}$, and $P_{p}=\bar{P}_{p}+P_{p c} P_{c}^{-1} P_{p c}^{\prime}$. Since $\bar{P}_{p}$ is symmetric definite positive and $P_{c}$ is symmetric, we get that $P_{p}$ is a symmetric definite matrix. Moreover, by Schur complement, the matrix $P=\left[\begin{array}{cc}P_{p} & P_{p c} \\ P_{p c}^{\prime} & P_{c}\end{array}\right]$ is symmetric definite positive, condition (18) holds, and we may apply Corollary 1 . From item 2 of Theorem 1 we know that the $x_{p}$ component of the solution converges to zero. As a first step of the proof, we will use the detectability assumption and the LaSalle invariance principle of Sanfelice et al. (2007) to prove next that also the $x_{c}$ component of the state converges to zero. To analyze the convergence properties of the solutions $x(\cdot, \cdot)=\left(x_{p}(\cdot, \cdot), x_{c}(\cdot, \cdot)\right): \operatorname{dom} x \rightarrow \mathbb{R}^{n_{p} \times n_{c}}$ from any initial condition, we consider two cases: i) assume that for some jump time $(t, j)$ (namely a hybrid time such that $(t, j) \in \operatorname{dom} x$ and $(t, j+1) \in$ $\operatorname{dom} x)$ we have $x_{p}(t, j)=0$. Then $x(t, j+1)=$ $\left(x_{p}(t, j), K_{p} x_{p}(t, j)\right)=0$ namely the solution $x(\cdot, \cdot)$ converges to zero in finite time.

ii) instead, if for all jump times $(t, j)$, we have $x_{p}(t, j) \neq$ 0 , then after each jump, the state necessarily belongs to the interior of the flow set $\bar{F}$. To see this, denote $x(t, j+1)$ by $x^{*+}$ and $x(t, j)$ by $x^{*}$ (possibly using the subscripts " $p$ " and " $c$ ") and note that from the assumption that $\overline{\widetilde{\alpha}} \leq \widetilde{\alpha}, x_{p}^{*} \neq 0$ and from (18) and (19b), we get

$$
\begin{aligned}
& \left(x^{*+}\right)^{\prime} N_{p} x^{*+}=\left[\begin{array}{c}
x_{p}^{*} \\
K_{p} x_{p}^{*}
\end{array}\right]^{\prime} \operatorname{He}\left(\left[\begin{array}{cc}
\bar{P}_{p} A_{p} & 0 \\
B_{p}^{\prime} \bar{P}_{p} & 0
\end{array}\right]\right)\left[\begin{array}{c}
x_{p}^{*} \\
K_{p} x_{p}^{*}
\end{array}\right] \\
& =\left(x_{p}^{*}\right)^{\prime} \operatorname{He}\left(\left[\begin{array}{c}
I \\
K_{p}
\end{array}\right]^{\prime}\left[\begin{array}{ll}
\bar{P}_{p} A_{p} & 0 \\
B_{p}^{\prime} \bar{P}_{p} & 0
\end{array}\right]\left[\begin{array}{c}
I \\
K_{p}
\end{array}\right]\right) x_{p}^{*} \\
& =\left(x_{p}^{*}\right)^{\prime} \operatorname{He}\left(\bar{P}_{p} A_{p}+\bar{P}_{p} B_{p} K_{p}\right) x_{p}^{*} \\
& <-\widetilde{\alpha}\left(x_{p}^{*}\right)^{\prime} \bar{P}_{p} x_{p}^{*} \leq-\overline{\widetilde{\alpha}}\left(x_{p}^{*+}\right)^{\prime} \bar{P}_{p} x_{p}^{*+},
\end{aligned}
$$

which, by the strict inequality at the last line, implies that $x^{*+}$ is in the interior of $\bar{F}$. Therefore, from continuity of the solutions satisfying the flow equation, after each jump the solution flows for some time $\bar{\tau}\left(x_{p}^{*}\right)$ before it reaches the boundary of the flow set (possibly jumping again). Define now $\tau_{m}=\min _{\left|x_{p}\right|=1} \bar{\tau}\left(x_{p}\right)$, which is a finite strictly positive scalar because the minimum is carried out over a compact set. Then, after each jump, the solution flows for at least $\tau_{m}$ times. Indeed, consider a generic jump to $x^{*+}=\left(x_{p}^{*}, K_{p} x_{p}^{*}\right)$ and note that, by homogeneity of the dynamics $(20)$, we can write $x(\tau, j+1)=\left|x_{p}^{*}\right| z(\tau, j+1)$ for all $\tau$ such that $(\tau, j+1) \in \operatorname{dom} x$, where $z(\cdot, j+1)$ is a suitable solution defined for all such values of $\tau$ and starting at $z(t, j+1)=\frac{1}{\left|x_{p}^{*}\right|}\left(x_{p}^{*}, K_{p} x_{p}^{*}\right)$. Then, by definition of $\tau_{m}$ and since $x_{p}^{*} \neq 0$ by assumption, the solution $z(\cdot, j+1)$ flows for at least $\tau_{m}$ times after $(t, j+1)$ and, consequently, so does the solution $x$.

Consider now the LaSalle theorem (Sanfelice et al., 2007, Corollary 4.4(b)) (see also (Goebel et al., 2009, Thm S13)) and apply it using the smooth function $V_{L S}(x)=V_{p}\left(x_{p}\right)$. By construction (see (19b)), the function $V_{L S}(\cdot)$ is non-increasing along flows for all $x$ in the flow set $\bar{F}$. Moreover, since $x_{p}$ remains constant along jumps, the function is also non-increasing along jumps for all $x$ in the jump set $\bar{J}$. Since we proved that there is a uniform lower bound $\tau_{m}$ on the elapsed time between any two jumps of the solutions under consideration, from (Sanfelice et al., 2007, Corollary 4.4(b)), all such solutions converge to the largest weakly invariant subset of the set where $V_{L S}(x)=V_{p}\left(x_{p}\right)=0$. Since $V_{p}\left(x_{p}\right)=0$ implies $x_{p}=0$, from the detectability condition on $\left(B_{p}, A_{c}\right)$ the largest weakly invariant subset reduces to the origin and this completes the proof that the $x_{c}$ component of the state converges to zero. 
By the above reasoning, we concluded that the state $x$ converges to zero. Then, since the dynamics is linear and the flow and jump sets are cones, we can apply (Goebel and Teel, 2010, Proposition 4.3) using $\omega(x)=|x|$, the standard dilation $M(\lambda)=\lambda$ and $d=1$. From this proposition the global convergence property implies global pre-asymptotic stability of the hybrid system which, in turns, implies global asymptotic stability by the completeness of all the trajectories. Finally, using once again the homogeneity property, the approach in (Nešić et al., 2011, Theorem 7) can be applied to conclude that global asymptotic stability implies global exponential stability.

Proof of Proposition 1. We start by proving the first part of the proposition. Consider any solution $\left(\bar{Q}_{p}, \rho_{y}, \rho_{x}, X\right)$ to the optimization problem (23). From the matrix inversion formula, denoting $\bar{P}_{p}=\left[\begin{array}{ll}P_{11} & P_{12} \\ P_{12}^{\prime} & P_{22}\end{array}\right]$ and recalling the notation for $\bar{Q}_{p}$ in the first inequality of (23), we have

$$
\begin{gathered}
P_{11}=\left(Q_{1}-\frac{q_{1 y} q_{1 y}^{\prime}}{q_{y}}\right)^{-1}, P_{22}=\frac{1}{q_{y}-q_{1 y}^{\prime} Q_{1}^{-1} q_{1 y}}, \\
P_{12}=-P_{22} Q_{1}^{-1} q_{1 y} .
\end{gathered}
$$

Let us inspect these terms separately. From the first constraint in (23), we have $\left[\begin{array}{cc}Q_{1} & q_{1 y} \\ q_{1 y}^{\prime} & q_{y}-1\end{array}\right]>\left[\begin{array}{ll}I & 0 \\ 0 & 0\end{array}\right] \geq 0$, which, by Schur complements, implies $Q_{1}-\frac{q_{1 y} q_{1 y}^{\prime}}{q_{y}-1}>0$ and $q_{y}-1-q_{1 y}^{\prime} Q_{1}^{-1} q_{1 y}>0$. These last two inequalities, together with $q_{y}>1$ and the last constraint of (23) imply respectively

$$
\begin{gathered}
q_{1 y} q_{1 y}^{\prime}<\left(q_{y}-1\right) Q_{1}<\rho_{y} Q_{1}, \\
0<q_{1 y}^{\prime} Q_{1}^{-1} q_{1 y}<q_{y}-1<\rho_{y}
\end{gathered}
$$

From (31) we get

$$
Q_{1}-\frac{q_{1 y} q_{1 y}^{\prime}}{q_{y}}>Q_{1}-\frac{\rho_{y}}{q_{y}} Q_{1}=\left(1-\frac{\rho_{y}}{q_{y}}\right) Q_{1}
$$

where we have used $q_{y}>1$ (from the first constraint of (23)) in the last step. By Schur complement applied to the fourth constraint of (23) and also using the fifth constraint of (23) we have

$$
Q_{1}>\rho_{x} \geq \frac{1}{\rho_{y}}
$$

Now consider a sequence of solutions $\left(\bar{Q}_{p}^{k}, \rho_{y}^{k}, \rho_{x}^{k}, X^{k}\right)_{k \in \mathbb{N}}$ to (23) such that $\rho_{y}^{k} \rightarrow 0$, as given by the assumption of Theorem 1, and consider the computations of this proof for this sequence. With $(32)$ we have $1 \leq q_{y}^{k}<\rho_{y}^{k}+1$ and thus $q_{y}^{k} \rightarrow 1$ and $\frac{\rho_{y}^{k}}{q_{y}^{k}} \rightarrow 0$ as $k \rightarrow \infty$. Combining (33) and (34) implies $P_{11}^{k} \rightarrow 0$, as $k \rightarrow \infty$. Consider now (32) and note that, by definition of $P_{22}^{k}$, we have $\lim _{k \rightarrow \infty} \frac{1}{P_{22}^{k}}=\lim _{k \rightarrow \infty} q_{y}^{k}=1$ because from the first and the last constraints of $(23), 1<q_{y}<1+\rho_{y}$. Therefore $\lim _{k \rightarrow \infty} P_{22}^{k}=1$. Finally, since $\bar{P}_{p}>0, P_{11}^{k} \rightarrow 0$ and $P_{22}^{k} \rightarrow 1$, we have $P_{12}^{k} \rightarrow 0$ as $k \rightarrow \infty$. As a consequence, $\bar{P}_{p}^{k} \rightarrow\left[\begin{array}{ll}0 & 0 \\ 0 & I\end{array}\right]$, and, due to the observability canonical form of $(13), V_{p}^{k}\left(x_{p}\right) \rightarrow|y|^{2}$ as $k \rightarrow \infty$, for each $x_{p}$ in $\mathbb{R}^{n_{p}}$. This completes the proof of the first part of the proposition. As a next step, we prove that using $K_{p}=X \bar{Q}_{p}^{-1}$ and $\bar{P}_{p}=\bar{Q}_{p}^{-1}$ where $X$ and $\bar{Q}_{p}^{-1}$ satisfy (23), equation (18) is satisfied for a small enough $\widetilde{\alpha}$. This trivially follows from the strict inequality in the third constraint of (23).

Finally, items 1 and 2 in the last part of the proposition follow from applying, respectively, item 2 of Theorem 1 and Theorem 3.

\section{Conclusions}

The design problem of a hybrid stabilizing loop on a continuous-time control system has been considered. The arising closed-loop system mixes discrete and continuous dynamics depending on the value of a nonlinear function. This allows to guarantee the stability and/or a decreasing property of some positive definite function. Adding a hybrid loop can be also instrumental when reducing the overshoot of a scalar output of a linear control system. Some simulations highlighted the interest of the results. This work lets some questions open. It might be of interest to better quantify the overshoot improvement achieved by the algorithm of Section 3.2 and possibly relate it to the rise time, as done in Beker et al. (2001) for a specific example. Moreover, the case of output feedback laws may be interesting to address. In this direction, recent work Fichera et al. (2012a,b) investigates output feedback extensions of the state feedback approach presented here, by using a Luenberger observer and implementing the hybrid loops in feedback from the observed state.

\section{References}

Aangenent, W. H. T. M., Witvoet, G., Heemels, W. P. M. H., van de Molengraft, M. J. G., Steinbuch, M., 2010. Performance analysis of reset control systems. International Journal of Robust and Nonlinear Control 20 (11), 1213-1233.

Anta, A., Tabuada, P., 2010. To sample or not to sample: self-triggered control for nonlinear systems. IEEE Transactions on Automatic Control 55, 2030-2042.

Artstein, Z., 1983. Stabilization with relaxed controls. Nonlinear Anal. 7 (11), 1163-1173.

Beker, O., Hollot, C., Chait, Y., 2001. Plant with integrator: an example of reset control overcoming limi- 
tations of linear feedback. IEEE Transactions on Automatic Control 46 (11), 1797-1799.

Beker, O., Hollot, C., Chait, Y., 2004. Fundamental properties of reset control systems. Automatica 40 (6), 905-915.

Fichera, F., Prieur, C., Tarbouriech, S., Zaccarian, L., 2012a. Hybrid state-feedback loops based on a dwelltime logic. In: Proc. of 4th IFAC Conf. on Analysis and Design of Hybrid Systems (ADHS). Eindhoven, The Netherlands.

Fichera, F., Prieur, C., Tarbouriech, S., Zaccarian, L., 2012b. Improving the performance of linear systems by adding a hybrid loop: The output feedback case. In: Proc. of American Control Conference (ACC). Montréal, Canada.

Freeman, R. A., Kokotović, P. V., 1996. Inverse optimality in robust stabilization. SIAM J. Control Optim. 34 (4), 1365-1391.

Goebel, R., Sanfelice, R., Teel, A., 2009. Hybrid dynamical systems. IEEE Control Systems Magazine 29 (2), 28-93.

Goebel, R., Teel, A. R., 2010. Preasymptotic stability and homogeneous approximations of hybrid dynamical systems. SIAM Review 52 (1), 87-109.

Haddad, W. M., Chellaboina, V., Kablar, N. A., 2001. Non-linear impulsive dynamical systems. Part II: Stability of feedback interconnections and optimality. International Journal of Control 74 (17), 1659-1677.

Lazar, M., Heemels, W., 2009. Predictive control of hybrid systems: Input-to-state stability results for suboptimal solutions. Automatica 45 (1), 180-185.

Lin, Y., Sontag, E., 1991. A universal formula for stabilization with bounded controls. Systems \& Control Letters 16 (6), 393-397.

Nešić, D., Zaccarian, L., Teel, A. R., 2005. Stability properties of reset systems. In: IFAC World Congress. Prague, Czech Republic.

Nešić, D., Zaccarian, L., Teel, A. R., 2008. Stability properties of reset systems. Automatica 44 (8), 2019-2026.

Nešić, D., Teel, A., Zaccarian, L., 2011. Stability and performance of SISO control systems with First Order Reset Elements. IEEE Transactions on Automatic Control 56 (11), 2567-2582.

Pan, Z., Ezal, K., Krener, A., Kokotovic, P., 2001. Backstepping design with local optimality matching. IEEE Transactions on Automatic Control 46 (7), 1014-1027.

Prieur, C., Astolfi, A., 2003. Robust stabilization of chained systems via hybrid control. IEEE Transactions on Automatic Control 48 (10), 1768-1772.

Prieur, C., Tarbouriech, S., Zaccarian, L., September 2010. Guaranteed stability for nonlinear systems by means of a hybrid loop. In: IFAC Symp. on Nonlinear Control Systems. Bologna, Italy, pp. 72-77.

Prieur, C., Tarbouriech, S., Zaccarian, L., 2011. Improving the performance of linear systems by adding a hybrid loop. In: 18th IFAC World Congress. Milan, Italy, pp. 6301-6306.

Sanfelice, R., Goebel, R., Teel, A. R., 2007. Invariance principles for hybrid systems with connections to de- tectability and asymptotic stability. IEEE Transactions on Automatic Control 52 (12), 2282-2297.

Sanfelice, R., Teel, A., Goebel, R., Prieur, C., 2006. On the robustness to measurement noise and unmodeled dynamics of stability in hybrid systems. In: Proc. of American Control Conference (ACC). Minneapolis, Minnesota, pp. 4061-4066.

Zaccarian, L., Nešić, D., Teel, A. R., 2011. Analytical and numerical Lyapunov functions for SISO linear control systems with First Order Reset Elements. International Journal of Robust and Nonlinear Control 21 (10), 1134-1158. 